\title{
Deformable Dose Accumulation with Image Guided Radiotherapy for Final Dose Evaluation in Pelvic Cases
}

\author{
Yunfeng Cui ${ }^{1 *}$, Jonathan W. Piper ${ }^{2}$, Amy S. Harrison ${ }^{1}$, Timothy N. Showalter ${ }^{1}$, Yan Yu ${ }^{1}$, James M. Galvin ${ }^{1}$ and Ying Xiao ${ }^{1}$
}

${ }^{1}$ Jefferson Medical College of Thomas Jefferson University, Philadelphia, PA ${ }^{2}$ MIMvista Corporation, Cleveland, $\mathrm{OH}$

\begin{abstract}
This study aims at a method that employs deformable dose accumulation for the evaluation of the results of intensity-modulated radiation therapy (IMRT) in pelvic cases. Each fraction dose was derived from the planning dose through rigid transformation accounting for setup corrections. Daily cone-beam CT (CBCT) images were used to deform the daily fraction dose to a reference geometry, which in our study was the first fraction geometry. A commercial software system, MIMvista, was used to perform deformable image registration, dose deformation, and dose accumulation. The differences in distributions of accumulated dose and planned dose were observed. The application of deformable dose accumulation is particularly useful in final accumulated dose evaluation throughout radiotherapy treatment, especially for the cases where there are significant deformations of planning target volume (PTV) and organs at risk (OARs).
\end{abstract}

Keywords: Deformable dose accumulation, Registration, Dose evaluation

\section{Introduction}

Deformable image registration improves the soft tissue alignment between different image datasets where the organ motion or deformation exists. Using this technique, radiation dose distribution from one image geometry can be more accurately transformed to the other images [1]. Deformable dose accumulation is an emerging method to accumulate the radiation dose from each fraction and evaluate the final dose distribution from radiation therapy [2]. Studies have shown the accuracy of deformable image registration in medical images and the advantage of dose transformation based on it $[3,4]$. Deformable image registration is being incorporated into more commercial software systems to assist with this procedure.

In this study, we applied the deformable dose accumulation to pelvic cases treated with intensity-modulated radiation therapy (IMRT). A commercial software system, MIMvista (Version 4.2.2; MIMvista Corp., Cleveland, $\mathrm{OH}$ ), was used. The differences between accumulated dose with deformation and the dose from planning were compared to demonstrate the application of this method in radiation dose evaluation.

\section{Material and Methods}

Two pelvic IMRT cases which were treated in our hospital were studied. The treatment target were prostate fossa and bladder respectively. The anatomy changes of the critical structures such as rectum, bladder, or small bowel and the planning target volume (PTV) were considered in these cases. The contours of the PTV and the organs at risk (OARs) were drawn on planning CT by physician. The CMS $\mathrm{XiO}$ (CMS Inc., St. Louis, MO) treatment planning system was used to generate IMRT plans for both cases. Daily on-line $\mathrm{kV}$ cone-beam CT (CBCT) images were taken using X-ray Volumetric Imager (XVI) of Elekta Synergy (Elekta Oncology Systems Ltd., Crawley, UK) treatment machine, to correct the patient setup through rigid alignment between planning CT and CBCT images. The planning CT, daily CBCT images, contours, and dose distribution calculated from treatment planning system were used to perform the deformable dose accumulation using the method described in the next section.

\section{Deformable dose accumulation}

Dose in each fraction was derived from planning dose by applying rigid transformation accounting for setup corrections. The tranformation map was from the rigid registration between planning $\mathrm{CT}$ and the daily CBCT. Registration was done using MIMvista software system and adjusted manually if necessary. The transfer of planning dose on planning CT to CBCT geometry includes the patient setup corrections with $\mathrm{CBCT}$ image guidance. The fraction doses on CBCTs were then deformed to a same reference geometry to allow deformable dose accumulation. The deformation map needed for this conversion was derived from the deformable image registration process. The reference geometry is the first fraction CBCT which was used to accumulate the dose since it is taken the closest in time as the planning $\mathrm{CT}$ and the software system has better performance when using the same modality images for deformable registration [5]. The deformable image registration was done between $\mathrm{CBCT}$ from first fraction and the CBCTs from other fractions. Finally, the fraction doses on reference geometry (i.e. the first $\mathrm{CBCT}$ geometry in this case) were added up to get the accumulated dose. The schematic diagram of the method is shown in Figure 1.

\section{Validation of deformable image registration}

The accuracy of deformable image registration has to be validated since the reliability of dose accumulation result is highly dependent on it. In this study, some typical anatomical points were identified in both images before the registration. These anatomical points should be chosen so as they are easy to identify with high confidence and

*Corresponding author: Yunfeng Cui, Division of Medical Physics, Dept. of Radiation Oncology, Thomas Jefferson University Hospital, Philadelphia,USA, E-mail: cyyan7@gmail.com

Received November 20, 2011; Accepted November 22, 2011; Published November 25, 2011

Citation: Cui Y, Piper JW, Harrison AS, Showalter TN, Yu Y, et al. (2011) Deformable Dose Accumulation with Image Guided Radiotherapy for Final Dose Evaluation in Pelvic Cases. J Nucl Med Radiat Ther S3:e001. doi:10.4172/2155-9619.S3-e001

Copyright: @ 2011 Cui Y, et al. This is an open-access article distributed under the terms of the Creative Commons Attribution License, which permits unrestricted use, distribution, and reproduction in any medium, provided the original author and source are credited. 
Citation: Cui Y, Piper JW, Harrison AS, Showalter TN, Yu Y, et al. (2011) Deformable Dose Accumulation with Image Guided Radiotherapy for Final Dose Evaluation in Pelvic Cases. J Nucl Med Radiat Ther S3:e001. doi:10.4172/2155-9619.S3-e001

minimum variability, so that the corresponding points in two images represent exactly same anatomical locations. After deformable image registration between the two images, those marker points in the secondary image are moved with deformation and should match the corresponding points in the reference image. By comparing the overlap of these pre-identified anatomical points, the registration result is validated in some extent.

\section{Dose distribution evaluation}

The dose distributions from deformable accumulation and direct accumulation (the first fraction dose multiplied by the number of fractions) were compared in terms of isodose lines and dose-volume histograms (DVHs). The contours used to generate DVH information included PTV and OARs which were derived from rigid transformation from the planning CT, with possible manual modifications.

\section{Results and Discussion}

Figure 2 shows an example of deformable image registration validation with an anatomical point chosen at the superior tip of left femoral head. The points were marked with pink cross both in reference CBCT image (Figure 1a) and secondery CBCT image (Figure $2 \mathrm{~b}$ ) from the prostate fossa case. Before image registration, the two points showed

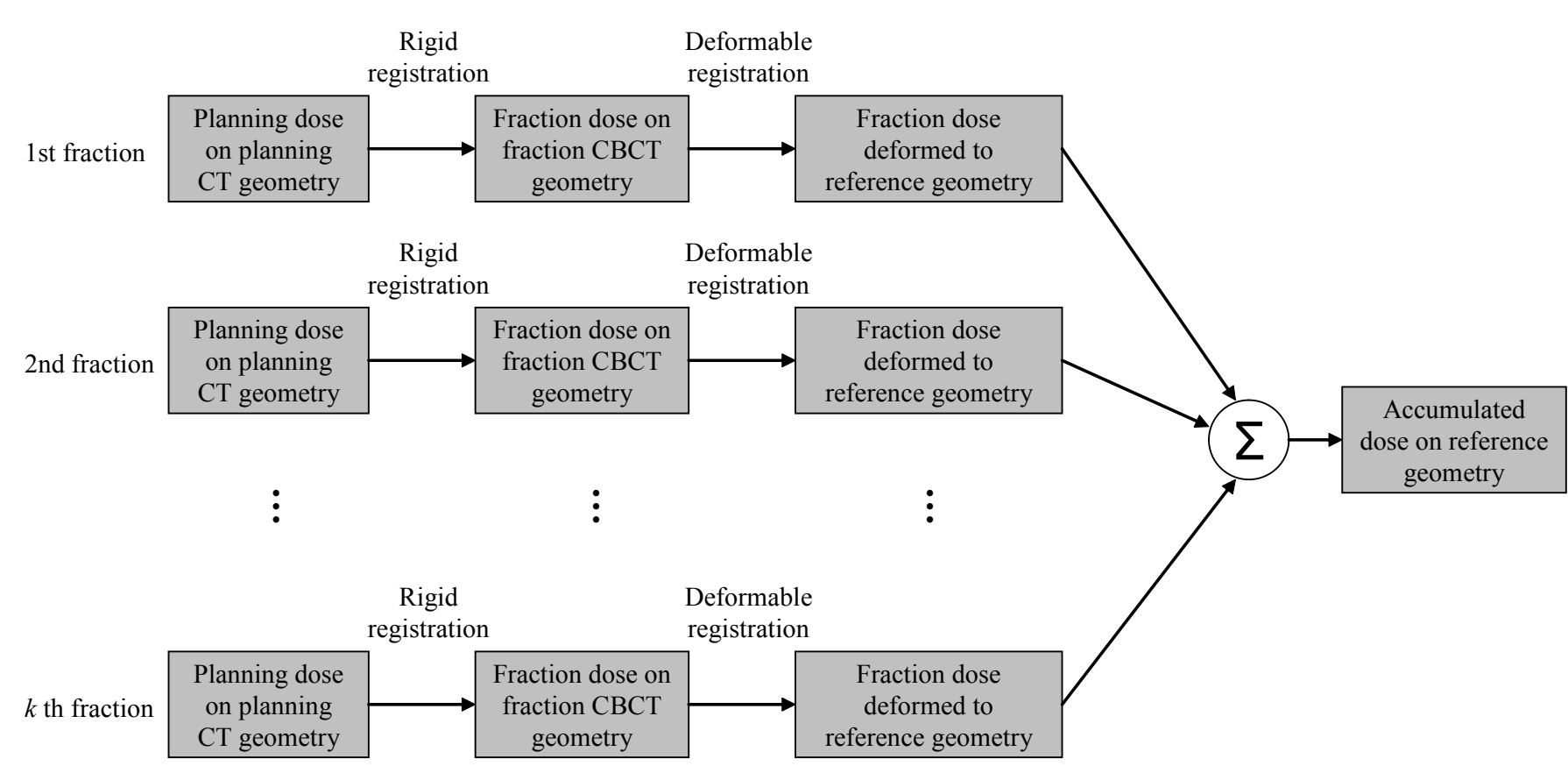

Figure 1: Schematic diagram of the method. $k$ : Number of fractions.

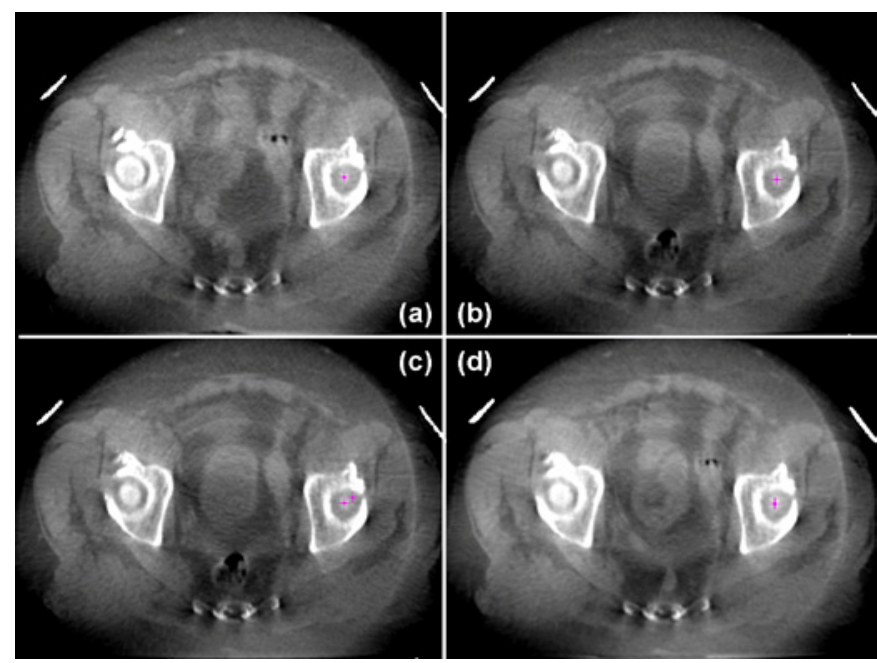

Figure 2: Validation of deformable image registration between two CBCTs from the prostate fossa case. (a) Reference CBCT image with the tip of left femoral head marked (pink cross). (b) Sencondary CBCT image with the tip of left femoral head marked (pink cross). (c) The distance between the selected anatomical points before image registration. Points are overlaid on the secondary CBCT image. (d) Secondary image after deformable registration to the reference image, showing excellent match between two pink points. 
Citation: Cui Y, Piper JW, Harrison AS, Showalter TN, Yu Y, et al. (2011) Deformable Dose Accumulation with Image Guided Radiotherapy for Final Dose Evaluation in Pelvic Cases. J Nucl Med Radiat Ther S3:e001. doi:10.4172/2155-9619.S3-e001

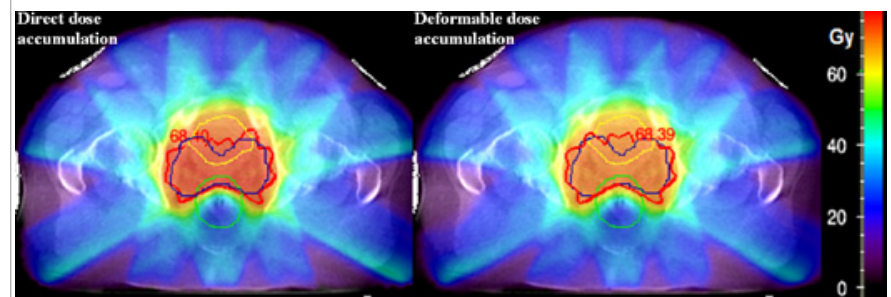

Figure 3: Dose distributions from direct dose accumulation (left image) and deformable dose accumulation (right image) on an axial slice of the prostate fossa case. Three contours shown in the images are PTV (dark blue), bladder (yellow), and rectum (green). Red isodose line is for the prescription dose of 6840cGy.

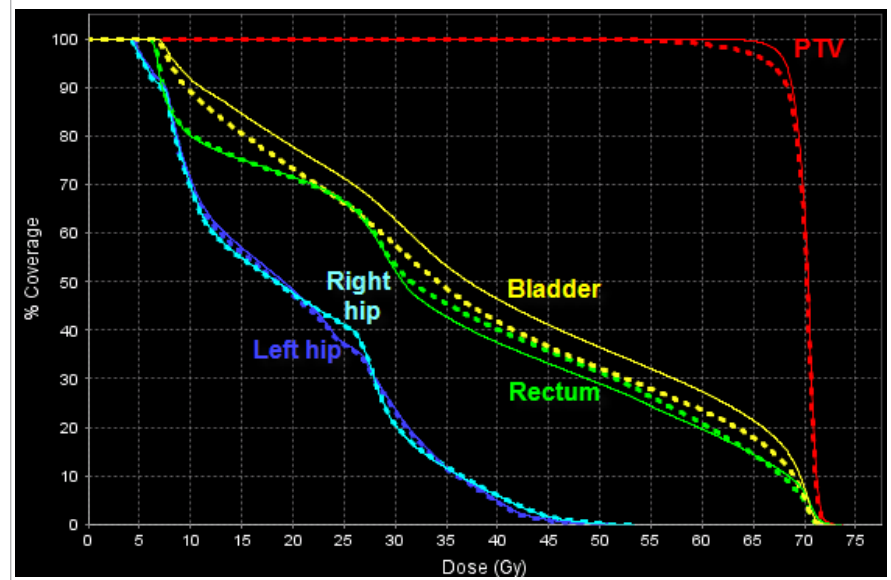

Figure 4: DVH for PTV and OARs derived from direct dose accumulation (solid lines) and deformable dose accumulation (dotted lines) in the prostate fossa case.

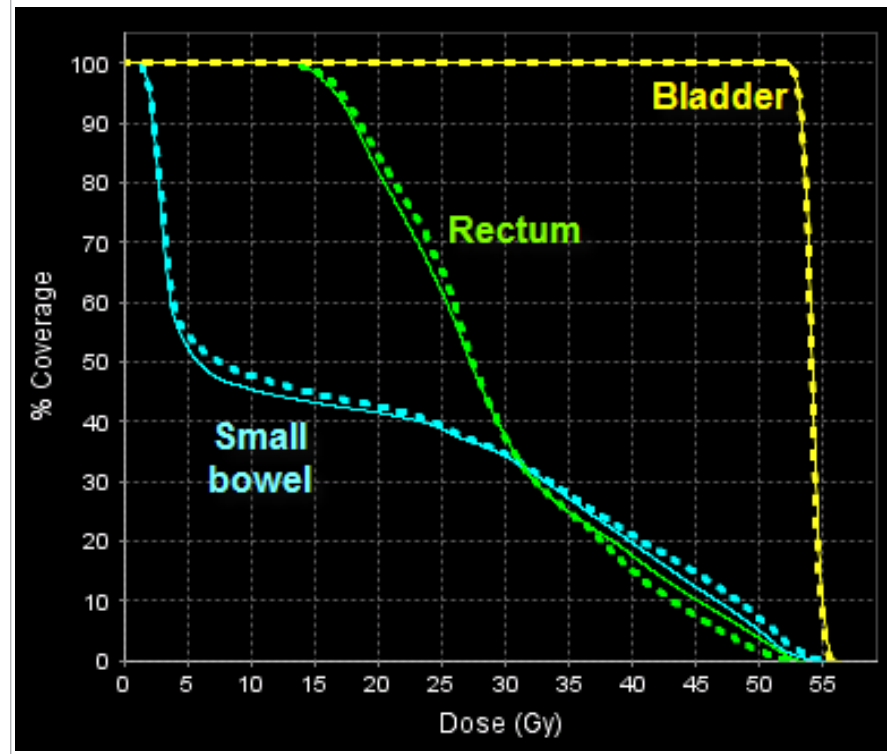

Figure 5: DVH for target and OARs derived from direct dose accumulation (solid lines) and deformable dose accumulation (dotted lines) in the bladder case.

more than $8 \mathrm{~mm}$ distance when overlaid together on the secondary CBCT image (Figure 1c). After deformable image registration, the secondary CBCT image were deformed to the reference image (Figure 1d) and the marked anatomical points in deformed secondary image and in reference image showed good agreement with less than 0.5 $\mathrm{mm}$ distance (Figure 1d). It is also worth noting that the image after registration (Figure 1d) showed significant change in bladder and rectum shapes, distinguishing the deformable transformation from rigid alignment. The other anatomical points used for validation of image registration also showed an alignment accuracy of less than 2 $\mathrm{mm}$ in this study.

Figure 3 shows an example of the dose distribution differences displayed on an axial slice from the prostate fossa case. The left image represents the dose distribution from direct accumulation and the right image is for deformable dose accumulation displayed on the same slice. Three contours displayed are PTV (dark blue), bladder (yellow), and rectum (green). The isodose line (red) for the prescription dose (6840cGy) is also shown in both images to demonstrate differences.

The DVH curves for the PTV and OARs of the prostate fossa case are shown in Figure 4. Histograms calculated from direct dose accumulation (solid lines) and deformable dose accumulation (dotted lines) are plotted together for comparison. It can be seen that there is almost no difference in DVH curves for left and right hips where positional changes are expected to be minimal. In contrast, the DVHs for structures that are less rigid show differences when the deformable dose accumulation was used. PTV coverage by the prescription dose (6840cGy) decreased from $93.7 \%$ (with direct dose accumulation) to $88.9 \%$ (with deformable dose accumulation). The V45Gy (the percentage volume that receives more than $45 \mathrm{~Gy}$ ) for bladder and rectum were $41.1 \%$ and $33.1 \%$ respectively with direct dose accumulation and changed to $36.6 \%$ and $35.7 \%$ with deformable accumulation. Figure 5 shows the DVH for a different site in the pelvis (bladder). The figure shows small differences for all DVH curves.

\section{Conclusions}

A method of deformable dose accumulation with daily $\mathrm{kV}$ CBCT with a software system was described in this study. The application of this method for two pelvic IMRT cases showed its benefit in the evaluation of accumulated dose distribution.

\section{Acknowledgement}

Yunfeng Cui: No conflict of interest; Jonathan W. Piper: Ownership interest and employment with MIMvista Software Inc.; Amy S. Harrison: No conflict of interest Timothy N. Showalter: No conflict of interest; Yan Yu: No conflict of interest; James M. Galvin: No conflict of interest; Ying Xiao: No conflict of interest.

\section{References}

1. Orban de Xivry J, Castadot P, Janssens G, Lee JA, Geets X, et al. (2010) Evaluation of the radiobiological impact of anatomic modifications during radiation therapy for head and neck cancer: can we simply summate the dose? Radiother Onco 96: 131-138.

2. Olivera G, Lu W, Ruchala K, et al. (2005) Image Guided Radiotherapy Plan Evaluation based on Deformable Dose Registration from Daily On-line Positioning Images. International Journal of Radiation Oncology Biology Physics 63 Supplement 1: S508-S509.

3. Janssens G, de Xivry JO, Fekkes S, Dekker A, Macq B, et al. (2009) Evaluation of nonrigid registration models for interfraction dose accumulation in radiotherapy. Med Phys 36: 4268-4276.

4. Brock KK (2010) Results of a Multi-Institution Deformable Registration Accuracy Study (MIDRAS). Int J of Radiat Oncol Biol Phys 76: 583-596.

5. Piper JW (2007) Evaluation of An Intensity-Based Free-form Deformable Registration Algorithm. Medical Physics 34: 2353-2354. 